\title{
A MATHEMATICAL ANALYSIS OF THE EVOLUTION OF HUMAN MATE CHOICE TRAITS: IMPLICATIONS FOR EVOLUTIONARY PSYCHOLOGISTS
}

\author{
NED KOCK ${ }^{*}$ \\ Texas A\&M International University
}

\begin{abstract}
Sexually reproducing organisms often rely on various traits to judge the attractiveness of potential mates. Many mate choice preferences and traits have evolved through selection by those organisms' ancestors, with traits having been either costly (detrimental to survival) or noncostly in the environment of their evolutionary adaptation. A general mathematical analysis of the evolution of traits used in mate choice is presented. The analysis builds on a combination of Price's covariance equation and Wright's method of path analysis, and includes a set of Monte Carlo simulations. The usefulness of the mathematical analysis is demonstrated through the development of a small but important set of hypotheses and implications for the human species: (1) costly traits used in mate choice by humans should be generally less common and more attractive to the other sex than non-costly traits; (2) costly traits should be disproportionately less common in human females than in males; and (3) some harmful human mental disorders, such as schizophrenia, may have co-evolved as costs of attractive mental traits. It is also shown that similar analyses can be easily employed by evolutionary psychologists to theorize about the evolution of complex mate choice traits, and to test the resulting theories with modern humans through the method of path analysis.
\end{abstract}

Keywords: Price equation, path modeling, path analysis, sexual selection, mate choice, costly traits, mental traits, mental disorders, brain evolution

\section{INTRODUCTION}

Evolved traits used in mate choice (Buss 1995; COHEN and BELSKY 2008; MiLleR 2000) can be costly or non-costly. Costly traits are often referred to as handicaps, and non-costly traits as indices (LYLE and SULLIVAN 2007; MAYNARD SMITH 1998; MAYNARD SMITH and HARPER 2003; ZAHAVI 1975, 2003; ZAHAVI and ZAHAVI 1997). The term "costly", when applied to a trait, means that the trait has a negative

* Address for correspondence: NeD Kock, Division of International Bus. and Tech. Studies, Texas A\&M International University, 5201 University Boulevard, Laredo, TX, 78041, USA, Tel: +1-956-326-2521, Fax: +1-956-326-2494;

Email: nedkock@gmail.com, Web page: http: //nedkock.com 
impact on survival. Costly traits used in mate choice are defined as traits that increase mating success while at the same time decrease survival success. Non-costly traits used in mate choice are defined as traits that increase mating success and that do not have a negative effect on survival success.

The train (often and erroneously referred to as the "tail") displayed by the male of the peacock species is an example of costly trait used in mate choice (ZAHAVI and ZAHAVI 1997). Having large trains with numerous eye spots significantly increases the mating success of male peacocks, while at the same time making them more vulnerable to predators (PETRIE et al. 1991; ZAHAVI and ZAHAVI 1997). A related behavioral trait is the propensity to display the train to females in leks during the mating season, without which the train itself would not be of much use.

The ability and motivation of males of the fruit fly species Drosophila subobscura to engage in the species' rapid courtship dance are examples of non-costly traits used in mate choice (MAYNARD SMITH and HARPER 2003). Males that show the ability and motivation to dance quickly and vigorously, in response to lead movements by females, increase their mating success. Those traits have no known negative impact on the survival success of males (MAYNARD SMITH and HARPER 2003).

Both costly and non-costly traits used in mate choice are generally assumed, at their initial stages of evolution, to reflect the existence of underlying traits that increase survival success, primarily resistance against parasites (HAMILTON and ZUK 1982; MAYNARD SMITH and HARPER 2003; MilleR 2000; WILSON 2000). However, since preferences and traits usually co-evolve, an increase in the frequency of a preference for a trait in a population often leads to a concomitant exaggeration of the trait, to the point where the trait starts presenting a survival cost for those individuals that display it (FISHER 1930; KOKKO et al. 2002). This is a different point than the one made in the paragraph above, and both points are consistent with one another, even though they may appear contradictory at first glance. That is, a mate choice trait may or may not have a survival cost, even though it reflects an underlying trait that was associated at early evolutionary stages with a survival advantage, such as resistance to parasites.

It has been argued that traits used in mate choice must be costly to be reliable (IREDALE et al. 2008; ZAHAVI 1975, 2003; ZAHAVI and ZAHAVI 1997). GRAFEN (1990) has shown that costly traits are indeed useful in mate choice, primarily because they are hard to fake, but not that only costly traits are reliable. If traits used in mate choice had to be costly to be reliable, it is unclear why costly mate choice traits seem to be much less common in nature than non-costly ones (DARWIN 1871; MAYNARD SMITH 1994; ZAHAVI and ZAHAVI 1997). The male peacock, for example, has a greater number of visible non-costly mate choice traits, such as the crest atop its head and the brightly colored feathers on its chest, than costly ones (of which the only obvious one is the train). It is also puzzling that costly traits are predominantly observed in males if they are so important for honest signaling. Males also choose females for mating. 
Human males and females appear to employ costly and non-costly traits for mate choice (Buss 1995; GANGESTAD and THORNHILl 2003; Millar 2010). A hypothesized costly mate choice trait displayed by males and used by females in mate choice is the presence of facial features indicating high levels of testosterone (e.g., angular facial features), apparently because testosterone suppresses the immune system and thus is an indicator that the males in question have had a particularly effective immune system up until the time they reached reproductive maturity (GANGESTAD and THORNHILl 2003; RHODES et al. 2003; THORNHILL and GANGESTAD 2006). Non-costly mate choice traits also seem to be more common than costly traits in both human males and females; examples are height and upper-body musculature in males, and hip-to-waist ratio and skin condition in females (Buss 1995, 1999; CARTWRIGHT 2000; FINK et al. 2001; FuRnHAM et al. 2006; MiLLER 2000; SHACKELFORD et al. 2005; SINGH and RANDALL 2007).

The discussion presented here aims at shedding some light on the issues above through a mathematical analysis of the evolution of costly and non-costly traits. The results of the analysis have important implications for evolutionary psychologists, which are also discussed. The analysis builds on a combination of the Price Equation (PRICE 1970) with the path analysis method developed by WRIGHT (1934, 1960), whereby a fundamental requirement for evolution of traits through selection is derived. The requirement is stated in terms of the covariance between the standardized measures of fitness and traits.

\section{A MATHEMATICAL ANALYSIS OF THE EVOLUTION OF MATE CHOICE TRAITS IN HUMANS}

The general mathematical analysis presented here applies to the vast majority of sexually reproducing organisms where mate choice takes place. Several important conclusions and implications for evolutionary psychologists are later derived from the mathematical analysis. A few exploratory hypotheses are also developed later in the paper, following directly from the mathematical analysis.

\section{The fundamental requirement for evolution of traits through selection}

For any subpopulation (e.g., males of a sexually reproducing species) of a population of organisms (males and females) to evolve phenotypic traits related to their fitness, the variation in the frequency of each trait in that subpopulation must satisfy Equation (1) in terms of its relation with fitness. This is the famous Price Equation (PRICE 1970), developed by George R. Price, who was for a short time a collaborator of notable evolutionary theorists William D. Hamilton and John Maynard Smith (FRANK 1995).

$$
\bar{W} \cdot \Delta \bar{Z}=\operatorname{Cov}(W, Z)+E(W \cdot \Delta Z)
$$


The term $Z$ in Equation (1) refers to a numeric variable tracking the presence or absence in an individual in the subpopulation of a particular phenotypic trait that has a genetic basis (e.g., $Z=1$ for presence of the trait, and $Z=0$ for absence). The fitness $W$ of an individual, which is defined as the number of surviving offspring of the individual, is assumed to be a linear function of each trait (FRANK 1997; RICE 2004).

The Price Equation has over the years become one of the most widely used algebraic formulations in evolutionary theorizing addressing a range of phenomena, from the evolution of morphological traits in various animals to the evolution of behavioral traits in humans (FLETCHER and ZWICK 2007; FRANK 1995, 1997; GRAFEN 2002, 2006; HENRICH 2004; MCELREATH and BOYD 2007; PAGE and NowAK 2002; VAN VEELEN 2005).

The covariance term in the Price Equation generally refers to the change due to differential survival and/or mating success conferred by a trait, while the expectation term refers to change due to other factors such as mutation and recombination (MCELREATH and Boyd 2007; RICE 2004). Therefore, the expectation term is usually set to zero when one wants to isolate the effects of environmental and sexual selection in the mathematical analysis of the likely evolution of a trait, and the comparative analysis of its evolution in relation with other traits. This leads to Equation (2), which is a simplified version of the Price Equation; the most widely used form in the analysis of the evolution of traits through selection (FRANK 1995, 1997; RICE 2004).

$$
\bar{W} \cdot \Delta \bar{Z}=\operatorname{Cov}(W, Z)
$$

The standardized equivalent of $W$ can be obtained by subtracting its mean from it and dividing the result by the standard deviation of $W$. The same can be done for $Z$, leading to two dimensionless quantities that are the standardized equivalents of $W$ and $Z$. These dimensionless quantities are referred to as $w$ and $z$. Expressing $W$ and $Z$ in terms of $w$ and $z$ in the covariance term of Equation (2), and transforming it using covariance properties leads to Equation (3).

$$
\begin{gathered}
\bar{W} \cdot \Delta \bar{Z}=\operatorname{Cov}\left(w \cdot S_{w}+\bar{W}, z \cdot S_{z}+\bar{Z}\right)=\operatorname{Cov}\left(w \cdot S_{w}, z \cdot S_{z}\right)= \\
S_{w} \cdot S_{z} \cdot \operatorname{Cov}(w, z) \Rightarrow \bar{W} \cdot \Delta \bar{Z}=S_{w} \cdot S_{z} \cdot \operatorname{Cov}(w, z) .
\end{gathered}
$$

The product on the left side of Equation (3) will always be positive for any trait that is undergoing evolution in a subpopulation. The same is true for the product of the standard deviations of $W$ and $Z$ on the right side of the equation, as long as the covariance term is greater than zero. Therefore, the inequality expressed in Equation (4) defines the fundamental requirement for the evolution of any fitnessrelated trait through selection: 


$$
\operatorname{Cov}(w, z)>0 \text {. }
$$

As will be seen in the following sections, Equation (4) will prove particularly useful because it is expressed in terms of the standardized variables $w$ and $z$, instead of the related non-standardized variables $W$ and $Z$. The main reason for this is that Equation (4) can be used in the context of path modeling and analysis, two well developed methods for the analysis of complex relationships between variables that build on properties of standardized variables.

\section{The evolution of independent traits used in mate choice}

FISHER (1930) has shown that in any sexually reproducing population, mate choice traits generally co-evolve with preferences for the traits. A preference must appear first in the population for the trait to be considered attractive, and thus evolve, whether it is costly or non-costly at any stage of the evolutionary process. KOKKO et al. (2002) have cogently shown that there is no reason to assume that selection necessarily favors preferences for either costly or non-costly traits. That is, what essentially matters from an evolutionary standpoint is the net contribution of the preference-trait combination to fitness, which is the reason why the probability of evolution of costly traits may be greater than zero. Thus the discussion presented here focuses on the evolution of mate choice traits, and assumes that preferences already exist for those traits. Moreover, it is assumed here that both preferences and traits appear stochastically in populations, either evolving in or being eliminated from those populations.

The discussion presented here builds on path analysis. This is a statistical analysis method that has been developed by Sewall Wright, one of the founders of the field of population genetics (DUNCAN 1966; KENNY 1979; MUELLER 1996; WRIGHT 1934, 1960). It relies on the development of path models, which are diagrams that facilitate the visualization of the relationships between variables measuring phenotypic traits, any intermediate variables, and fitness (RICE 2004).

Figure 1 shows a path model with two standardized variables quantifying phenotypic traits of individuals in a subpopulation, $x$ and $y$, where the traits are independent from each other. Error terms have been left out of the path model for simplicity. Both traits measured through $x$ and $y$ have positive effects on mating success $(m)$, which is defined as the standardized measure of the total number of copulations in which an individual has participated during his or her lifetime. These effects are indicated as $p_{m x}$ and $p_{m y}$, and are due to $x$ and $y$ being traits used in mate choice; that is, the traits are perceived as attractive by individuals of the other sex. Trait $y$ also has a negative effect on survival success (s), which is the standardized measure of the age of an individual at the time of death. This effect is indicated as $p_{s y}$. 


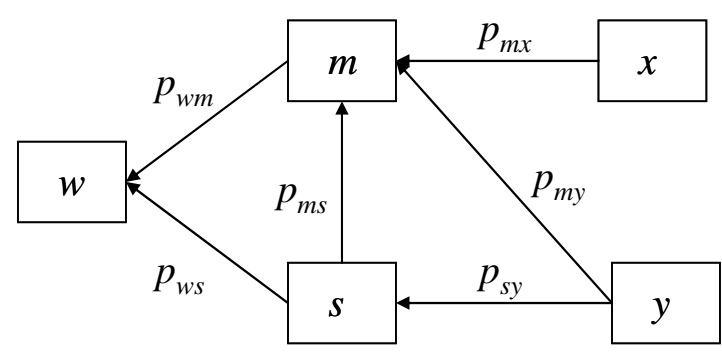

Figure 1. Path model showing costly $(y)$ and non-costly $(x)$ mate choice traits

Both $m$ and $s$ have positive effects on the standardized measure of fitness ( $w$ ), indicated in Figure 1 as $p_{w m}$ and $p_{w s}$. Since an individual must be alive to have a copulation, it follows that $s$ has a positive effect on $m$. All effects $\left(p_{m x}, p_{m y}\right.$, etc.) are measured as standardized partial regression coefficients, also referred to as path coefficients. They are of the same type as the coefficients generated in multiple regression analyses (MUELLER 1996; WRIGHT 1960).

A fundamental property of path models is that the covariance between any pair of variables equals the sum of the products of the path coefficients in all possible paths connecting the two variables (DUNCAN 1966; MuELLER 1996; WRIGHT 1934, 1960). Combining this property with Equation (4) for the trait $x$ leads to the requirement for evolution of that trait expressed in Equation (5).

$$
p_{w m} \cdot p_{m x}>0 \Rightarrow p_{m x}>0 .
$$

Equation (5) essentially states that if the effect of $x$ on $m$ is positive, then the trait measured by $x$ will tend to evolve, because the effect of $m$ on $w$ is always positive. Or, in other words, if a mutation evolves in the individuals of the sex that is selecting a non-costly trait measured by $x$, where that mutation makes those individuals find the trait attractive, then the trait will tend to evolve. Equation (6) is derived in a similar way, but this time focusing on $y$ :

$p_{w m} \cdot p_{m y}+p_{w s} \cdot p_{s y}+p_{w m} \cdot p_{m s} \cdot p_{s y}>0 \Rightarrow p_{w m} \cdot p_{m y}>-p_{s y} \cdot\left(p_{w s}+p_{w m} \cdot p_{m s}\right) \Rightarrow$

$$
p_{m y}>-p_{s y} \cdot\left(\frac{p_{w s}}{p_{w m}}+p_{m s}\right) .
$$

A comparison of Equations (5) and (6) reveals one important property, which is that the evolution of a costly trait $y$ requires $p_{m y}$ to be above a certain threshold. The threshold is represented by the product on the right side of Equation (6). There is no such threshold for the evolution of a non-costly trait $x$. This property is explicitly formalized in Equations (7) and (8), where the threshold is referred to as $\hat{p}_{m y}$ : 


$$
\begin{gathered}
\hat{p}_{m y}=-p_{s y} \cdot\left(\frac{p_{w s}}{p_{w m}}+p_{m s}\right), \\
p_{m y}>\hat{p}_{m y} .
\end{gathered}
$$

A few important conclusions can be derived from the above discussion. The probability of evolution of a costly trait will be lower than the probability of evolution of a non-costly trait, because of the existence of a threshold that any costly trait will have to overcome to evolve. Also, more often than not the effect of costly traits on mating success (measured through $p_{m y}$ ), namely the level of attractiveness of costly traits, will be higher than that of non-costly traits.

Focusing now on Equation (7), it is clear that the threshold for evolution of a non-costly trait is proportional to the absolute value of the negative effect of the trait on survival success $\left(p_{s y}\right)$ and to the term $p_{w s} / p_{w m}+p_{m s}$. Therefore the magnitude of a costly trait's positive effect on mating success $\left(p_{m y}\right)$, which could be seen as the level of attractiveness of the trait, will be proportional to the negative impact of the trait on survival success. That is, the more costly a trait used in mate choice is, the more attractive it becomes.

The term $p_{w s} / p_{w m}+p_{m s}$ is particularly relevant for the discussion presented here because of the potentially high impact that one of its component terms, the ratio $p_{w s} / p_{w m}$, can have on the threshold for evolution of $y$ traits and also the magnitude of the effect of those traits on mating success. Equations (9) and (10), derived from path model properties (DUNCAN 1966; WRIGHT 1934, 1960), show how $p_{w s}$ and $p_{w m}$ can be calculated from $r_{w s}, r_{w m}$ and $r_{m s}$; these are the Pearson correlation coefficients between $w$ and $s, w$ and $m$ and $m$ and $s$, respectively:

$$
\begin{aligned}
& p_{w m}=\frac{r_{w m}-r_{w s} \cdot r_{m s}}{1-r_{m s}{ }^{2}}, \\
& p_{w s}=\frac{r_{w s}-r_{w m} \cdot r_{m s}}{1-r_{m s}{ }^{2}} .
\end{aligned}
$$

Life history theory (ANDERSON 1990; HILL and KAPLAN 1999; KOKKO 1998; LANDE 1982) provides a body of empirical findings and related theoretical conclusions that can be used in the understanding of the relationships between $s, m$ and $w$, and their likely correlations $\left(r_{w s}, r_{w m}\right.$ and $\left.r_{m s}\right)$ in many species, including the human species. (Life history theory is not only a theory, but an area of research in biology.) Among the conclusions that can be reached based on life history theory, the following are particularly relevant for the arguments advanced here.

- The value of $r_{w s}$ will be generally high in a population of individuals when the level of offspring dependence on parents for survival to reproductive 
age is high; and more broadly when the level of parental investment required for raising offspring to reproductive age is high.

- The value of $r_{w m}$ will be generally low when there are factors hindering targeted copulation, such as ovulation being at least partially concealed and/or the use of fertility-cycle-dependent mating strategies (e.g., short- and longterm strategies); and when survival of offspring to reproductive age is highly dependent on factors other than number of copulations (e.g., parental care).

- The value of $r_{m s}$ will be generally low when reproductive maturity is reached relatively late in life; and when mating success is lightly dependent on factors other than survival success, which would be the case in a balanced-sexratio population of individuals with a tendency toward monogamy.

Life history theory allows for the expectation of high values for $r_{w s}$ and moderate to low values for $r_{w m}$ and $r_{m s}$ in certain species, such as modern humans and their ancestors during the Pleistocene. The Pleistocene is the period in which much of the evolution of the genus Homo is believed to have taken place, leading up to the emergence of Homo sapiens (BOAZ and ALMQUIST 2001). It goes from approximately 1.8 million to 11 thousand years ago.

From Equations (9) and (10) it can be seen that small differences between the correlation coefficients $r_{w s}$ and $r_{w m}$ can be amplified as differences between the path coefficients $p_{w s}$ and $p_{w m}$ even though the absolute values of the path coefficients are reduced. This in turn leads to high $p_{w s} / p_{w m}$ ratios, and thus to high thresholds for evolution of $y$ traits.

Let us take for example the case in which $r_{w s}=.35, r_{w m}=.25$ and $r_{m s}=.25$. This would lead to $p_{w s}=.31, p_{w m}=.17$ and $p_{w s} / p_{w m}=1.77$. If we increased $r_{w s}$ to .45 and kept both $r_{w m}$ and $r_{m s}$ at .25 then the ratio $p_{w s} / p_{w m}$ would become 2.82. Let us assume that a non-costly trait $x$ evolved with a $p_{m x}$ of .25 under these latter conditions, and a costly trait $y$ with a $p_{s y}$ (negative effect on survival success) of -.15 also evolved. That costly trait would have at a minimum a $p_{m y}$ of .42 to have evolved, which would make it significantly more attractive to the selecting sex than the trait $x$. That is, $\left(p_{m y}=.42\right)>\left(p_{m x}=.25\right)$.

\section{Does the path model assume linear relationships, and does it reflect what happens at the individual level?}

At this point two fundamental questions must be addressed to clarify the nature of the relationships depicted by the path model. The first question is: Does the path model assume linear relationships? This is an important question because most relationships between variables describing natural phenomena are nonlinear (O'MEARA 2000; WOLD et al. 2001), and there is no reason to believe that this is a modern phenomenon. The second question is: Does the path model reflect what happens at the individual level? This is also an important question because the path model and 
related equations appear to break down at the individual level; e.g., an individual whose mating success is zero cannot leave offspring, and thus should have zero fitness, even though there is an alternative fitness-enhancing path in the model via survival success.

Does the path model assume linear relationships? The answer is no. As noted by RICE (2004), the "covariance greater than zero" requirement for evolution, summarized earlier as the fundamental requirement for the evolution of any fitnessrelated trait through selection, has a very specific meaning. It states that the linear association between trait and fitness must be positive, whether the underlying relationship is linear or nonlinear. The reason is that the covariance between two standardized variables equals the correlation between the variables (HAIR et al. 2009), and the correlation is a measure of linear association (ROSENTHAL and ROSNOW 1991). This is a point that was also stressed by HAMILTON and ZUK (1982) in their discussion of the evolution of mate choice traits. For example, let us assume that the relationship between a trait and fitness is logarithmic. The fundamental requirement for the evolution of the trait is still that it must be positively correlated with fitness, regardless of the logarithmic shape of the relationship (see also, MCELREATH and BOYD 2007).

Does the path model reflect what happens at the individual level? The answer is also no, because evolution is a population phenomenon. Strictly speaking, genotypes or individuals do not evolve; only populations evolve (GILLESPIE 2004; HARTL and ClARK 2007; LiEBERMAN et al. 2005). Evolution refers to a process whereby a genotype appears in a population (in one or more individuals) and increases in frequency in the population, generation after generation, because the genotype codes for a phenotypic trait that confers a fitness advantage to those individuals that possess it. When this happens, it is frequently said that the genotype, and the trait it codes for, are "evolving" in the population.

A more correct assertion would have been that "the population is evolving" in regards to the genotype or trait. If the genotype codes for a trait that offers no fitness advantage, the genotype will not increase in frequency in the population via selection. The path model does not describe what happens at the individual level, it describes what happens in the population. A positive path coefficient between two variables reflects an underlying positive linear association between the two variables. Not all individuals in a population must express a trait for a positive linear association to exist between the trait and fitness, or between the trait and intermediate variables. Similarly, not all individuals in a population must mate for a positive association between mating success and fitness to exist; and not all individuals must survive to reproductive maturity for a positive association between survival success and fitness to exist. 


\section{The limits on the number of independent traits used in mate choice}

Unlike traits evolved because of their differential effect on survival success, which are caused by genetic mutations that are expected to quickly spread through a population and thus lose much of their variability, traits used in mate choice usually retain a great deal of variability over time (HAMILTON and ZUK 1982; KOKKO et al. 2002; LEHMANN et al. 2007; MAYNARD SMITH 1971, 1998). Because of this, mate selection traits take up a proportion of the explained variance of $m$, which in path models cannot be higher than 1 (MUELLER 1996; WRIGHT 1960), effectively crowding the variance space that can be occupied by other mate choice traits. That is, as more independent mate selection traits are evolved, the probability that new such traits will evolve gradually decreases until it reaches zero. This does not mean that mutations that led to new traits will not appear in individuals in the subpopulation; they might, but they will not evolve, meaning that their frequency in the subpopulation will not increase. Their effect on fitness will be effectively zero, characterizing them as neutral mutations; at least until selective pressures change and "reawaken" them, so to speak.

Figure 2 illustrates a situation where there are multiple independent traits used in mate choice. Costly traits are indicated as $y 1, y 2 \ldots y u$. Non-costly traits are indicated as $x 1, x 2 \ldots x k$. A relevant question in this situation is whether there is a limit on the number of such traits that can evolve. The answer is yes, and builds on an-

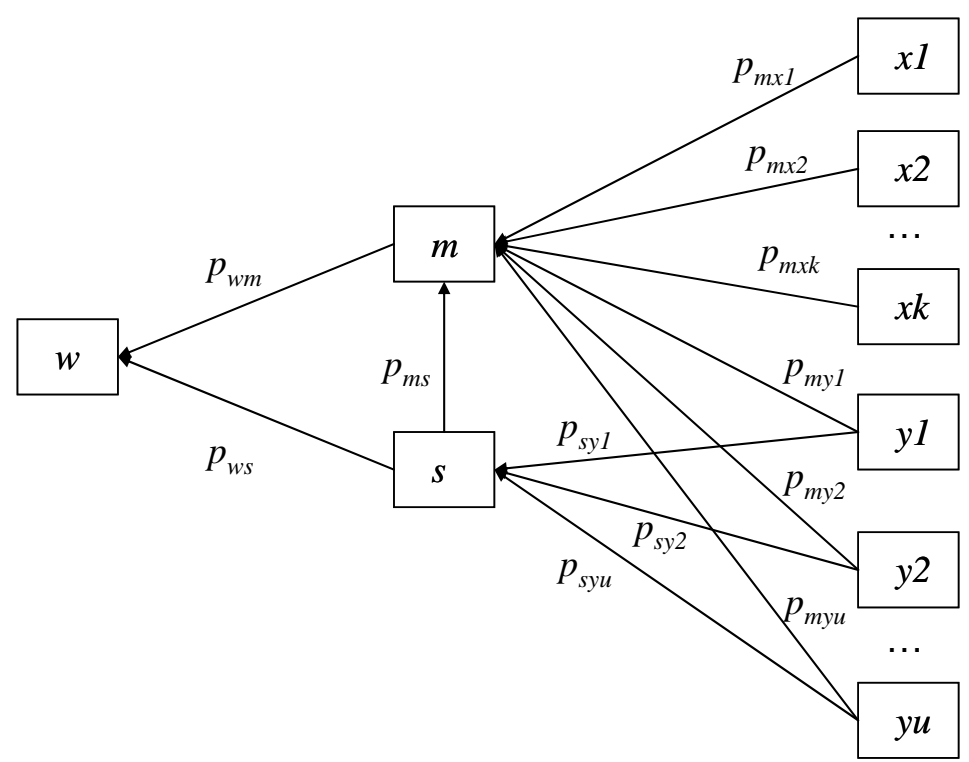

Figure 2. Path model with costly $(y 1, y 2 \ldots y u)$ and non-costly $(x 1, x 2 \ldots x k)$ mate choice traits 
other property of path models briefly discussed earlier: the sum of the products of the path coefficients and related correlations associated with the paths pointing at each endogenous variable cannot be greater than 1 (DUNCAN 1966; WRIGHT 1934, 1960).

The sums of products of path coefficients and correlations associated with variables pointing at each endogenous variable in the path model shown in Figure 2 , as well as the property above related to each of those endogenous variables (that those sums of products cannot be greater than 1), are indicated in Equations (11) to (16). For simplicity error terms are not indicated. Unless those error terms are zero, the sums of products are always lower than 1 . The endogenous variables in question are $w, s$, and $m$. The sums of products associated with those variables are indicated as $R_{w}{ }^{2}, R_{s}{ }^{2}$ and $R_{m}{ }^{2}$, respectively. Equations (11) and (12) are derived from Equations (9) and (10), where the path coefficients are represented as functions of the correlation coefficients among $w, s$, and $m$ :

$$
\begin{gathered}
R_{w}{ }^{2}=p_{w m} \cdot r_{w m}+p_{w s} \cdot r_{w s} \Rightarrow R_{w}{ }^{2}=\frac{r_{w m}{ }^{2}+r_{w s}{ }^{2}-2 \cdot r_{w m} \cdot r_{w s} \cdot r_{m s}}{1-r_{m s}{ }^{2}}, \\
R_{w}{ }^{2} \leq 1 .
\end{gathered}
$$

Since all traits $y 1, y 2 \ldots y u$ are assumed to be independent from each other, their path coefficients to the variable $s$ equal their respective correlation coefficients. This yields Equations (13) and (14):

$$
\begin{gathered}
R_{s}{ }^{2}=r_{s y 1}{ }^{2}+r_{s y 2}{ }^{2}+\ldots+r_{s y u}{ }^{2}, \\
R_{s}{ }^{2} \leq 1 .
\end{gathered}
$$

As with traits $y 1, y 2 \ldots y u$, the traits $x 1, x 2 \ldots x k$ are also assumed to be independent from each other and from the $y$ traits. Therefore all of their path coefficients to the variable $m$ equal their respective correlation coefficients. This leads to Equations (15) and (16):

$$
\begin{gathered}
R_{m}{ }^{2}=p_{m s} \cdot r_{m s}+\left(r_{m \times 1}{ }^{2}+r_{m \times 2}{ }^{2}+\ldots+r_{m x k}{ }^{2}\right)+\left(r_{m y 1}{ }^{2}+r_{m y 2}{ }^{2}+\ldots+r_{m y y}{ }^{2}\right), \\
R_{m}{ }^{2} \leq 1
\end{gathered}
$$

As can be seen from Equations (11) to (16) the bottleneck for the evolution of multiple independent costly and non-costly traits is $R_{m}{ }^{2}$. Equation (11) shows that $R_{w}{ }^{2}$ is independent from the effects of the traits on $m$ and $s$. Moreover, a compari- 
son of Equations (15) and (13) leads to the conclusion that $R_{m}{ }^{2}$ is normally significantly greater than $R_{s}{ }^{2}$ for any number of traits of types $x$ and $y$, since for all traits $y 1, y 2 \ldots y u$ the following conditions usually must be satisfied if those traits are to evolve: $r_{m y 1}>r_{s y 1}, r_{m y 2}>r_{s y 2} \ldots r_{m y 3}>r_{s y 3}$.

To illustrate the limitation on the possible number of independent traits used in mate choice, let us assume various non-costly traits with $r_{m \times 1}=r_{m \times 2}=\ldots=r_{m \times k}=$ .25 . Since costly traits have generally larger effects on $m$, let us also assume various costly traits with $r_{m y 1}=r_{m y 2}=\ldots=r_{m y u}=.35$. Finally, let us assume that non-costly traits are 3 times as likely to evolve as costly traits. (This is consistent with the results of Monte Carlo simulations discussed later.) Given these conditions, a maximum of 12 traits used in mate choice could possibly evolve, 9 of which would be non-costly and 3 would be costly. Such a small number of traits is inconsistent with the wide variety of mate choice traits actually employed by various animals species, including humans (BUSS 1995, 1999; CARTWRIGHT 2000; FINK et al. 2001; FURNHAM et al. 2006; GANGESTAD and THORNHILl 2003; MAYNARD SMITH and HARPER 2003; MiLLER 2000; RHODES et al. 2003; SHACKELFORD et al. 2005; SINGH and RANDALL 2007; THORNHILL and GANGESTAD 2006; ZAHAVI 1975, 2003; ZAHAVI and ZAHAVI 1997).

Independent traits can evolve under certain conditions, such as when the traits are influenced by one or a few non-pleiotropic genes in different loci of different chromosomes, and no epistatic interactions exist between genes coding for different traits (GILLESPIE 2004; MAYNARD SMITH 1998). However, given that traits used in mate choice usually reflect body resistance to parasites that are (i.e., the parasites) constantly evolving at a high speed, evolution should favor a high variety of such mate choice traits (HAMILTON and ZUK 1982; KOKKO et al. 2002). This can only be achieved if the traits present interdependencies; that is, if the measures of some of the traits are correlated with one another. From a mathematical perspective, the reason for this is that those interdependencies would generally reduce the values of the path coefficients between the traits and $m$, and thus allow for more traits used in mate choice to coexist.

Consistently with the above discussion, there are many traits used in mate choice among humans, and several show interdependencies. Examples of traits used in mate choice whose measures seem to be correlated in human males are facial indicators of testosterone levels (angular features), height, and upper-body muscular mass (BUSS 1995; MILLER 2000), as well as general intelligence and creative intelligence (HASELTON and Miller 2006; Miller 2000b). Given that one key argument made here is that costly traits used in mate choice are generally more attractive than non-costly ones, we now need to consider whether this is the case when costly and non-costly traits show interdependencies. This is examined in the next section through a comparison of the threshold for evolution of costly and non-costly traits whose measures are correlated. 


\section{The evolution of interdependent traits used in mate choice}

Figure 3 shows a path model identical to the one shown in Figure 1 with the only difference being in the curved arrows connecting the traits $x$ and $y$, where $x$ is a noncostly trait used in mate choice and $y$ is a costly one. The curved arrow indicates that a level of covariance exists between the measures of traits $x$ and $y$, which is equivalent to saying that those measures are correlated.

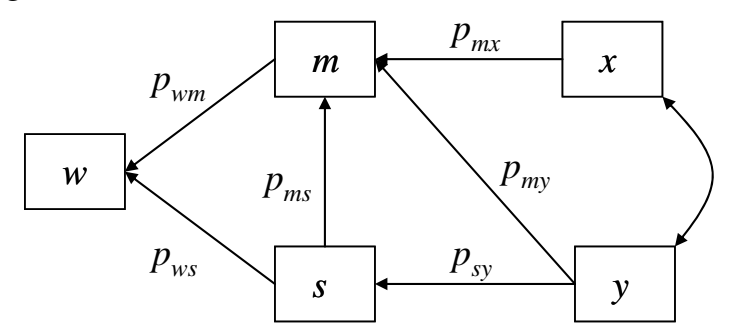

Figure 3. Path model showing interdependent costly $(y)$ and non-costly $(x)$ mate choice traits

We can use path analysis properties (MUELLER 1996; RICE 2004; WRIGHT $1934,1960)$ here in the same way as in the development of the inequalities that described the conditions for evolution of independent traits. Doing so leads to Equations (17) to (20). Equations (17) and (18), shown below, define the threshold and condition for evolution of a non-costly trait $x$ whose measure is correlated with that of a costly trait $y$ :

$$
\begin{gathered}
\hat{p}_{m x}=-p_{s y} \cdot p_{x y} \cdot\left(\frac{p_{w s}}{p_{w m}}+p_{m s}\right)-p_{m y} \cdot p_{x y}, \\
p_{m x}>\hat{p}_{m x .}
\end{gathered}
$$

Equations (19) and (20), shown below, similarly define the threshold and condition for evolution of a costly trait $y$ whose measure is correlated with that of a non-costly trait $x$ :

$$
\begin{gathered}
\hat{p}_{m y}=-p_{s y} \cdot\left(\frac{p_{w s}}{p_{w m}}+p_{m s}\right)-p_{m x} \cdot p_{x y}, \\
p_{m y}>\hat{p}_{m y} .
\end{gathered}
$$

Since $p_{x y}=r_{x y}$ it follows that the threshold for evolution of trait $y$ will almost always be higher than the threshold of evolution of the trait $x$. The difference between the two thresholds will tend to decrease as $r_{x y}$ increases, reaching very low values as $r_{x y}$ approaches 1 . (This is a theoretical point at which the standardized 
measures of the traits are statistically indistinguishable.) When $r_{x y}=0$ the difference between the thresholds is high. In this case, as expected, Equations (17) and (20) are reduced to the equivalent forms derived based on the assumption that the traits were independent from one another. That is, the right side of Equation (17) becomes zero and Equation (19) is reduced to Equation (7).

Equations (17) and (19) have two interesting features. One is that each threshold, for evolution of $x$ and $y$, now depends on the level attractiveness of the other trait (i.e., $p_{m x}$ and $p_{m y}$ ). The higher $p_{m x}$ is the lower is the threshold for evolution of $y$, and vice versa. The other interesting feature is that both thresholds can now assume negative values, thus making it easier for both costly and non-costly interdependent traits to evolve.

The addition of other interdependent traits significantly increases the complexity of the threshold equations, and the analysis of path models with any number of traits is beyond the scope of the arguments presented here. Nevertheless, two important conclusions can be reached based on the discussion so far. One conclusion is that the addition of new traits whose measures are correlated will generally lower the threshold for evolution of new costly and non-costly traits for mate choice. This is true whether the new traits are costly or non-costly. This effect will be counterbalanced by the crowding of the variance space caused by new traits being evolved. Another conclusion is that, as with independent traits, the more costly a trait used in mate choice is, the more attractive it is likely to be, even if its measure is correlated with that of one or more costly or non-costly traits.

\section{Probability of evolution and relative attractiveness of traits}

The probabilities discussed in this section were obtained from Monte Carlo simulations (ROBERT and CASELLA 2005) in which 1,000 cases were generated with independent random values for the path coefficients shown in the path models. In path models reflecting actual phenomena (including biological phenomena) rarely path coefficients between pairs of variables are higher than the correlations between the variables (MUELLER 1996; MARUYAMA 1998; WrighT 1934). Also, given the incremental phenotypic effect of most new mutations (BOAZ and ALMQUIST 2001; FOX and WOLF 2006; GILLESPIE 2004; MAYNARD SMITH 1998) path coefficients in models depicting traits and their intermediate effects on fitness can be reasonably expected to have values between 0 and .5. Therefore the path coefficients were made to vary randomly within that range (i.e., from 0 to .5) in the Monte Carlo simulations. For $p_{s y}$ the range of variation was from 0 to -.5 . Continuous uniform distributions were used.

The exceptions to the above rule of thumb used in the Monte Carlo simulations were the path coefficients $p_{w s}$ and $p_{w m}$, which appear in the threshold equations as part of the ratio $p_{w s} / p_{w m}$. Following the previous discussion based on life history theory (ANDERSON 1990; HILL and KAPLAN 1999; KOKKO 1998; LANDE 1982) and 
its application to species where survival success plays a more significant role in determining their fitness than mating success, such as in humans and their ancestors during the Pleistocene, that ratio can be conservatively assumed to vary between 1 and 3 for simulation purposes. Therefore the ratio $p_{w s} / p_{w m}$ was made to vary randomly within that range (i.e., from 1 to 3 ) in the Monte Carlo simulations.

Figure 4 shows the conditional probabilities of evolution of costly $(y)$ and noncostly $(x)$ traits used in mate choice, obtained from the Monte Carlo simulations, assuming that preferences for the traits had already appeared in the sex selecting the traits. (The assumption that the preferences had already spread in the sex selecting the traits is not necessary; they may have co-evolved with the traits.) The graph at the top shows the conditional probabilities for independent traits; the graph at the bottom shows the probabilities for traits that are assumed to be interdependent.
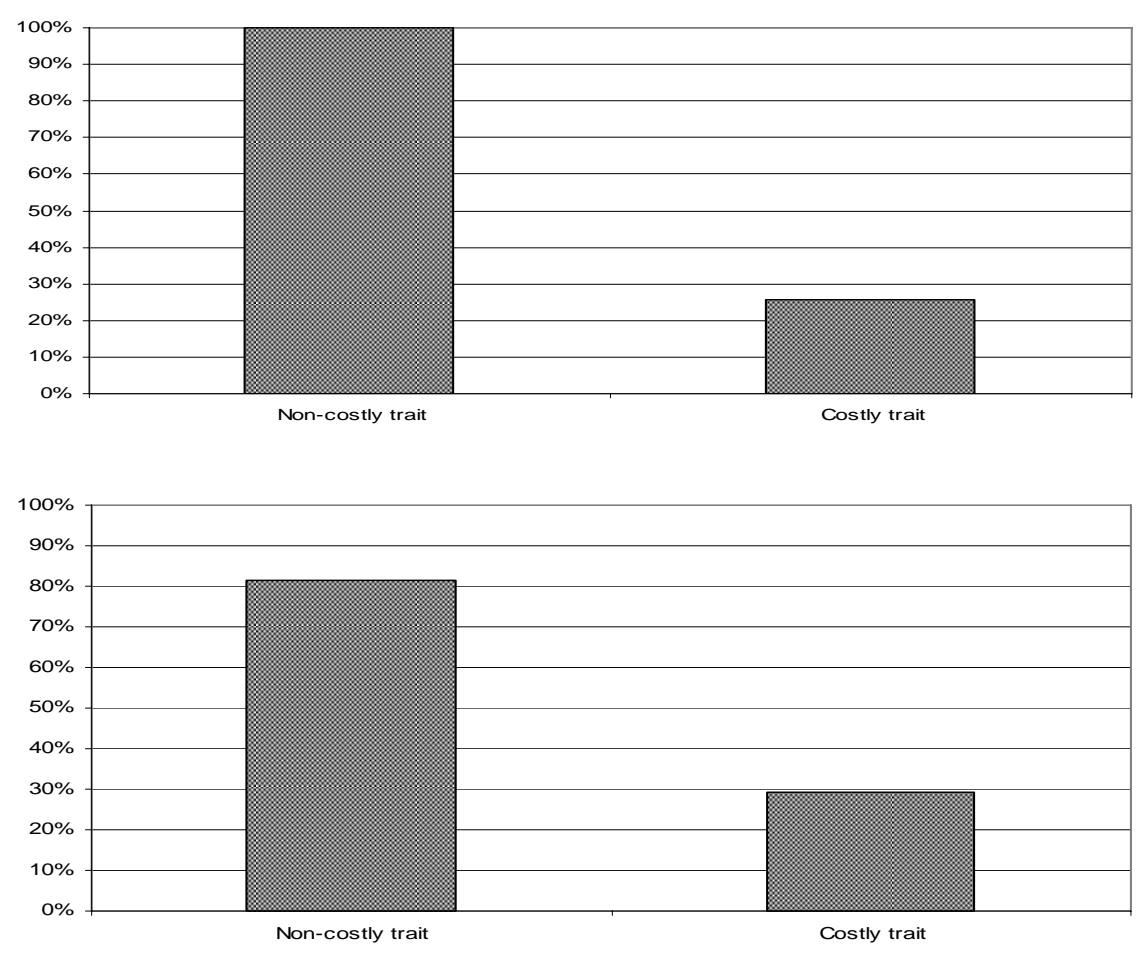

Notes:

- Top: Traits x and y are independent; bottom: traits are interdependent.

- Probabilities' conditions: preference for traits $\mathrm{x}$ and $\mathrm{y}$ existed in the sex using the trait for mate choice.

Figure 4. Conditional probabilities of evolution of costly $(y)$ and non-costly $(x)$ mate choice traits 
The conditional probabilities that an evolved costly $(y)$ trait will be more attractive than an evolved non-costly $(x)$ trait are shown in Figure 5. The two conditions used for the calculation of these probabilities were that: (a) preference for traits $x$ and $y$ existed (even if not in a widespread manner) in the sex using the trait for mate choice; and (b) the thresholds required for evolution of both traits were surpassed. The graphs show the probabilities as a function of the threshold for evolution of costly $(y)$ trait, which is generally the highest threshold of the two. The graph at the top assumes that the traits are independent; the graph at the bottom assumes the traits to be interdependent.
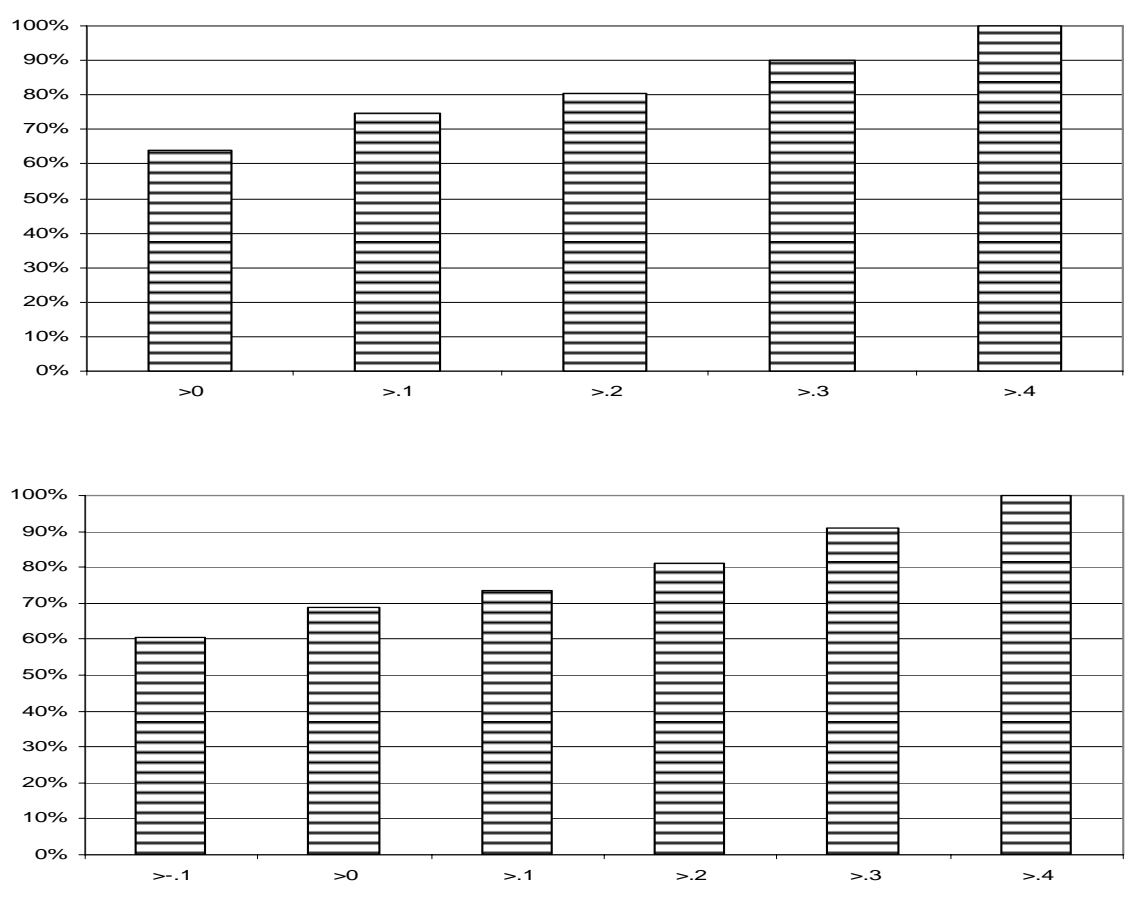

Notes:

- Top: Traits x and y are independent; bottom: traits are interdependent.

- Horizontal axis: threshold for evolution of trait $\mathrm{y}$.

- Probabilities' conditions: (a) preference for traits $\mathrm{x}$ and $\mathrm{y}$ existed in the sex using the trait for mate choice; (b) the thresholds for evolution of both traits were surpassed.

Figure 5. Conditional probabilities that a costly $(y)$ mate choice trait will be more attractive than a non-costly $(x)$ trait as a function of the threshold for evolution of costly $(y)$ trait

Figure 6 shows the ratios of mean attractiveness of costly $(y)$ and non-costly $(x)$ traits as a function of the threshold for evolution of costly $(y)$ trait. Those ratios 
can be represented as $\bar{p}_{m y} / \bar{p}_{m x}$ for each threshold range, and can be seen as measures of how much more attractive on average trait $y$ should be in comparison with trait $x$ for a given threshold range; e.g., a ratio of 1.5 means that trait $y$ is $50 \%$ more attractive on average than trait $x$.
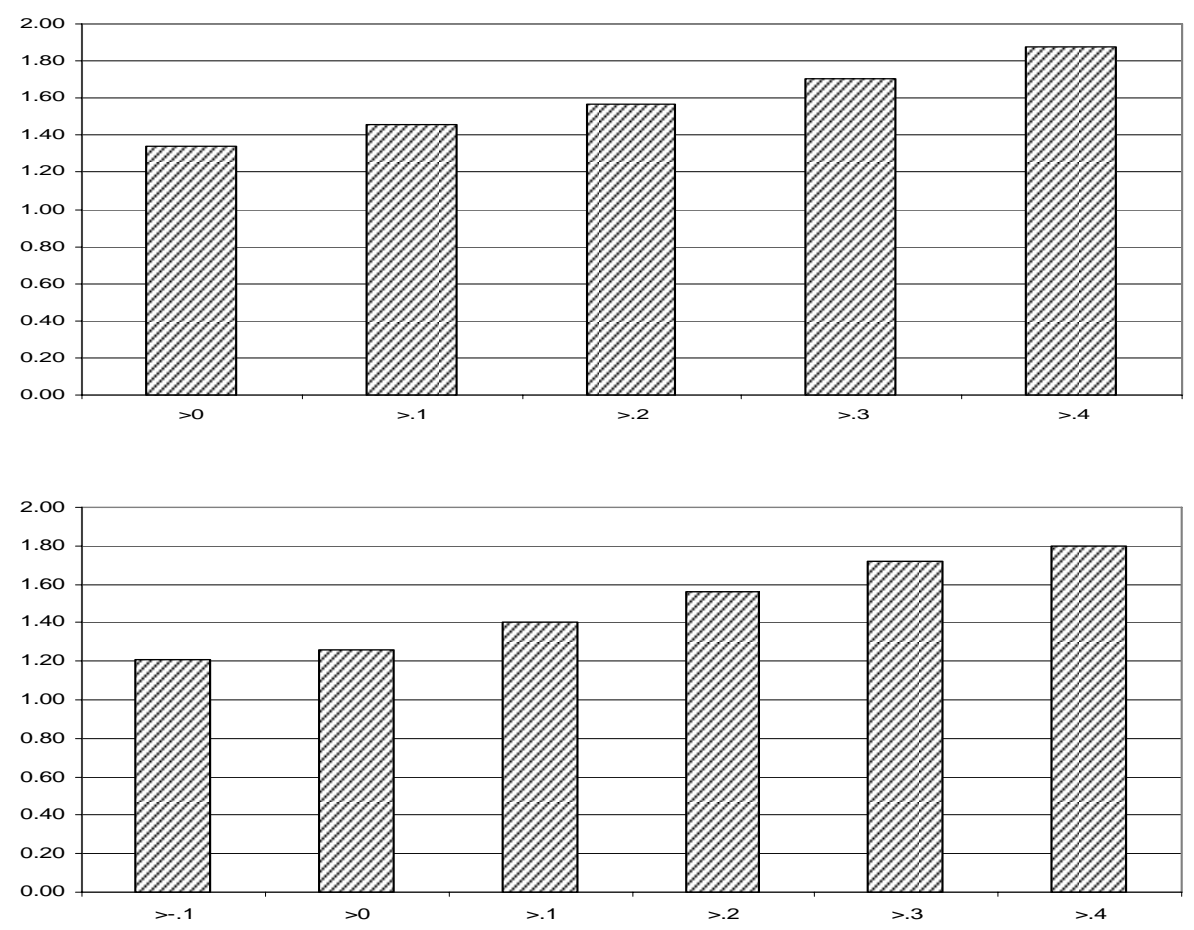

Notes:

- Bars show a measure of how much more attractive on average trait y should be in comparison with trait $\mathrm{x}$ for a given threshold; e.g., a ratio of 1.5 means that trait $\mathrm{y}$ is $50 \%$ more attractive on average than trait $\mathrm{x}$.

- Top: Traits $\mathrm{x}$ and y are independent; bottom: traits are interdependent.

- Horizontal axis: threshold for evolution of trait $\mathrm{y}$.

- Probabilities' conditions: (a) preference for traits $\mathrm{x}$ and $\mathrm{y}$ existed in the sex using the trait for mate choice; (b) the threshold for evolution of both traits have been surpassed.

Figure 6. Ratios of mean attractiveness of costly $(y)$ and non-costly $(x)$ mate choice traits as a function of the threshold for evolution of costly $(y)$ trait

In Figure 6, the graph at the top indicates the ratios of mean attractiveness for independent traits, and the graph at the bottom shows the ratios of mean attractiveness for traits that are assumed to be interdependent. Note that the leftmost threshold indicator for the graph at the bottom is -.1 in Figures 5 and 6, which reflects the fact that thresholds for interdependent traits can assume negative values. 


\section{WHAT DOES THIS ALL MEAN FOR EVOLUTIONARY PSYCHOLOGISTS?}

The mathematical analysis discussed above opens a number of avenues for future research in the field of evolutionary psychology. Among these, three are particularly important. The first refers to the use of path models similar to the ones discussed above to theorize about the evolution of complex mate choice traits (see, e.g., WELLS et al. 2009), which would probably involve more complex paths than the ones discussed above. The second refers to the use of a sophisticated multivariate analysis method, namely path analysis (DUNCAN 1966), to explore modified theoretical path models leading to predictions about psychological traits in modern humans. The third avenue for future research in the field of evolutionary psychology is direct exploratory theorizing based on the basic results already suggested by the mathematical analysis, which could form the basis for novel empirical investigations on evolved psychological traits. These three avenues are discussed below.

\section{Using path models to theorize about the evolution of complex mate choice traits}

The path models discussed so far are only a small subset of the models that can be developed by evolutionary psychologists to theorize about the various aspects of the evolution of mate choice traits in humans. Path modeling of the type employed in the mathematical analysis describes in a diagrammatic way the algebraic structure of the evolution of mate choice traits, as those traits spread or disappeared in ancestral populations.

Let us assume that an evolutionary psychologist has good reasons to believe that two psychological traits, one costly $(y)$ and the other non-costly $(x)$, have been caused by a precursor psychological trait $(a)$ in our ancestral past. This could lead to a model like the one in Figure 7, which includes the precursor trait $(a)$, and is thus more complex than a model incorporating only the two costly $(y)$ and non-costly $(x)$ traits.

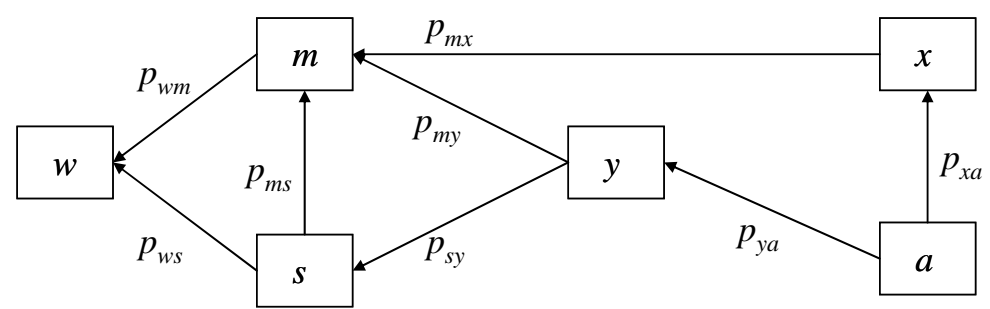

Figure 7. Example of more complex path model of evolution of mate choice traits 
Let us assume, for the sake of discussion, that the psychological traits depicted above were the following: openness to new thoughts $(a)$, degree of creative intelligence $(x)$, and degree of schizophrenia $(y)$. The model reflects a few theoretical expectations about the evolution of the traits among ancestral males, driven by female selection. Openness to new thoughts is hypothesized to positively influence the degrees of creative intelligence and of schizophrenia. The degree of schizophrenia of a male is hypothesized to negatively influence his mating success and survival success. That is, in this model both $p_{m y}$ and $p_{s y}$ are hypothesized to be negative. The degree of creative intelligence of a male is hypothesized to positively influence his mating success. These hypotheses could be explicitly represented in the model, through plus and minus signs, for completeness.

By developing such a model a researcher must explicitly theorize relationships between evolutionarily relevant variables. This is likely to help researchers avoid naïve (a.k.a. Panglossian) evolutionary theorizing, which appears to be common in the field of evolutionary psychology (CARTWRIGHT 2000); and also convince other researchers that their theorizing is not naïve (e.g., a "just so" theory). Moreover, the relationships carry a very specific mathematical meaning. This meaning is that, for the evolution of the traits $a$ and $x$ to have taken place among ancestral humans, the sums of the products of the path coefficients in all possible paths connecting the two variables and fitness must have been positive.

It should be stressed that path models do not have to include all possible variables to be used in theorizing, because path models invariably assume the existence of uncorrelated error. The uncorrelated error terms refer to "hidden" variables; that is, variables that are not explicitly included in a model. This would be reflected in the assumption that a model with a small number of variables would only explain a certain percentage of the variance in the variable fitness. Mathematically speaking, that percentage of explained variance is calculated as the R-squared statistic for the variable fitness (HAIR et al. 2009), which is unlikely to equal 1 for a model with a small number of variables. Nevertheless, such a model would still be invaluable for evolutionary psychological theorizing, as it makes explicit testable relationships between traits, intermediate variables, and fitness.

\section{Using path analysis to explore modified models for modern humans}

Each of the path coefficients in a path model that refers to ancestral humans is a standardized partial regression coefficient. This is exactly the type of coefficient generated by multiple regression analysis and path analysis (HAIR et al. 2009; KLINE 1998). Conceptually, multiple regression analysis is a special case of path analysis, as path models can be decomposed into simpler models, each with multiple independent variables pointing at a single dependent variable (i.e., multiple regression models). The same is true for most statistical tests employed in behavioral research, such as ANOVA and ANCOVA. Because these tests can be shown to be 
special cases of multiple regression analysis (RENCHER 1998; ROSENTHAL and RosNOw 1991), they can also be shown to be special cases of path analysis.

Therefore a path model that refers to ancestral humans can be used as a basis for an empirical test involving modern humans. The results of the empirical test involving modern humans would in turn either support or falsify the original model (i.e., the one that refers to ancestral humans). Certain elements of the original model should be removed prior to the test (see Figure 8). For example, the notion of fitness $(w)$ in our ancestral past may carry little meaning in an urban population of modern humans who control their fertility, in which case the variable $w$ should be removed. (This may not always be the case, such as in populations of modern isolated hunter-gatherers.) Survival success (s) may also be removed if the focus of the test is on the modern variable attractiveness, or a related variable (e.g., desirability as a short-term sex partner). The variable attractiveness, or a related variable, may replace, or be used as a surrogate for, mating success $(m)$.

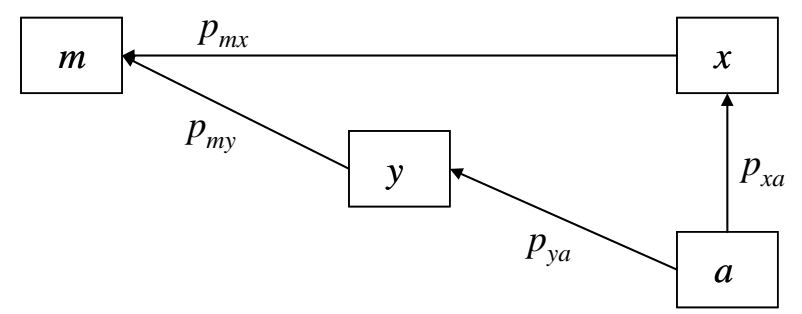

Figure 8. Example of mate choice path model to be tested with modern humans

The adaptation of an ancestral theoretical path model (i.e., that refers to ancestral humans) to produce a modern path model, as shown above, has two main advantages. One is that it allows for the retention of enough elements of the ancestral model to make it clear that the modern model tests evolved mate choice traits. The other advantage is that the analysis of the empirical data can employ any software tool that implements path analysis. Usually software tools that implement structural equation modeling, which is essentially path analysis with latent variables, also implement path analysis (KLINE 1998; MARUYAMA 1998). Examples of those software tools are Amos, LISREL, PLS-Graph, and WarpPLS (CHIN 2001; KLINE 1998; KOCK 2010; SCHUMACKER and LOMAX 2004).

\section{Exploratory theorizing based on the mathematical analysis}

The mathematical analysis discussed earlier opens up a third avenue for future research in the field of evolutionary psychology, in addition to avenues discussed in the preceding sections. This third avenue refers to direct exploratory theorizing 
based on the basic results already suggested by the mathematical analysis. Examples are provided in the following section and its subsections. These examples focus on the rarity and attractiveness of costly traits, frequency of occurrence of these traits in males and females, and undesirable side effects of costly traits.

\section{EXAMPLES OF EXPLORATORY THEORIZING BASED ON THE MATHEMATICAL ANALYSIS}

The exploratory hypotheses discussed below, developed based on the mathematical analysis, are essentially theoretical speculations that can be used as a basis for the design of future empirical studies. They are relevant for evolutionary psychologists investigating mate choice traits, as well as broader evolutionary psychological issues. Some of the hypotheses address longstanding issues in connection with the evolutionary basis of certain human traits, which are yet to be settled.

\section{Costly traits used in mate choice by humans should be generally less common and more attractive than non-costly ones}

Costly traits used in mate choice, whether they are independent of or dependent on non-costly mate choice traits, generally have a higher threshold to overcome in order to evolve than non-costly traits. That threshold is represented by the term $\hat{p}_{m y}$ in the equations, which is itself a function of the survival cost of each costly trait. Therefore, the probability of evolution of costly mate choice traits is generally lower than that of non-costly ones, which in turn suggests that costly mate choice traits should be less common in modern humans than non-costly traits. The Monte Carlo simulations (which are based on very conservative assumptions) suggest that the probability of evolution of a non-costly mate choice trait is approximately 4 times higher than that of an independent costly trait, and approximately 3 times higher than that of a costly trait whose measure is correlated with that of the noncostly trait.

Because of the higher threshold that they have to overcome in order to evolve, costly traits used in mate choice will be generally more attractive than non-costly traits, whether the costly and non-costly traits are independent or interdependent. The higher the threshold for evolution of a costly trait used in mate choice, the higher will also be: (a) the probability that the costly trait will be more attractive than a non-costly trait; and (b) the ratio of mean attractiveness of costly and noncostly traits. These of course will be true only if the costly trait evolves. The Monte Carlo simulations suggest that the probability that a costly trait will be more attractive than a non-costly trait will be $90 \%$ or higher for evolution threshold $\left(\hat{p}_{m y}\right)$ values of .3 and higher. In those cases, a costly mate choice trait is expected to be on 
average at least $70 \%$ more attractive than a non-costly trait. It is important to note that the threshold for evolution of a costly trait can be high even if the survival cost of the trait is relatively low. This will occur as long as the ratio $p_{w s} / p_{w m}$ is high.

The above conclusions are consistent with non-costly mate choice traits appearing to be much more common than costly traits in both human males and females. Some examples of non-costly traits are height and upper-body musculature in males, and hip-to-waist ratio and skin condition in females (BuSs 1995, 1999; CARTWRIGHT 2000; FINK et al. 2001; FURNHAM et al. 2006; Miller 2000; SHACKELFORD et al. 2005; SINGH and RANDALL 2007). The above conclusions are also consistent with creative intelligence being considered to be one of the most attractive mental traits in human males (HASELTON and MILLER 2006); creative intelligence seems to be strongly correlated with certain harmful mental disorders in humans, such as schizophrenia, which likely posed a significant survival handicap to our human ancestors (KELLER and MiLLER 2006; NESSE and WiLLIAMS 1994).

\section{Costly traits used in mate choice should be disproportionately less common in human females than males}

The previous discussion based on life history theory (ANDERSON 1990; HILL and KAPLAN 1999; KOKKO 1998; LANDE 1982) and its application to humans and their ancestors during the Pleistocene suggests that the ratio $p_{w s} / p_{w m}$ may have had significantly higher values for females than males. The ratio provides a measure of the relative effect of survival on fitness compared with the effect of mating on fitness. It is likely to increase as the survival of the individual to which the ratio refers becomes more important than his or her mating success in the generation of surviving offspring. Survival success is likely to be significantly more important than mating success in females, in terms of their effect on fitness.

The above conclusion was prominently acknowledged by both DARWIN (1871) and FISHER (1930) as being at the forefront of their thinking on sexual selection. It is also consistent with Bateman's principle, which states that females are a limiting resource in any population (BATEMAN 1948; HARTL and CLARK 2007). It follows from several key observations, including the following: (a) human females are the ones that produce eggs, and thus the number of healthy females is highly correlated with the number of surviving offspring produced by any population of humans (this also applies to the vast majority of animals); (b) since males are much less selective of their sexual partners than females, the survival success of human females is of much more importance for the number of surviving offspring than their mating success; and (c) human females are the ones that provide the most care to infants, particularly during the years the infants are most vulnerable to survival threats.

Based on a detailed analysis of viability and reproduction tables for various human groups, FISHER (1930) also noted that the survival success of ancestral female humans was so important as to justify infanticide (or severe infant neglect) 
under very poor environmental conditions (e.g., extreme scarcity of food), so that the females could maintain their health to bear children when the environmental conditions improved. That discussion was presented as part of FISHER's (1930) formulation of what he refers to as the Malthusian parameter of population increase, which relates the coefficients of survival and mating in one single equation that applies to various populations under different conditions.

As noted earlier, the ratio of the effects of survival and mating on fitness, represented by the term $p_{w s} / p_{w m}$ in the equations, has a disproportionately high impact on the probability of evolution of costly traits used in mate choice. The greater is the ratio, the lower is the probability of evolution of costly traits, with the probability quickly approaching zero as the term increases. For example, increasing the range of random variation of that term from 1 to 3 to the range 3 to 6 in the Monte Carlo simulations with interdependent traits makes the probability of evolution of a costly trait 3 times less likely (a reduction from approximately $30 \%$ to $10 \%$ ). Similar probability reductions are observed regardless of the costly trait's dependence on other costly or non-costly traits. Based on this and the above discussion, one can thus conclude that the term $p_{w s} / p_{w m}$ would likely have reached values in the Pleistocene that would have made costly traits used in mate choice much less likely to have evolved in ancestral females than in males, and thus much less common in modern human females than in males.

The above conclusion is consistent with costly mate choice traits being observed in human males but not in females. An example of costly trait displayed by men and found attractive by women is the presence of facial features indicating high levels of testosterone (e.g., angular facial features), since testosterone is hypothesized to suppress the immune system and thus act as a costly indicator of high immune system effectiveness (GANGESTAD and THORNHILL 2003; RHODES et al. 2003; THORNHILL and GANGESTAD 2006). There apparently are no such costly mate choice traits in human females (see, e.g., BuSs 1995).

\section{Certain harmful human mental disorders may have evolved as costs of attractive mental traits}

Certain harmful mental disorders in humans, such as schizophrenia, are often seen as puzzles from an evolutionary perspective. In some cases, the heritability of mental disorders and their frequency in the population at various levels of severity suggests that they may have been evolved through selection, yet they often significantly decrease the survival prospects of those afflicted by them (KELLER and MiLLER 2006; NESSE and WiLLIAMS 1994). The question often asked is why have they evolved at all? Should not they have been eliminated, instead of maintained, by selective forces? The discussion in the previous sections allows for the conclusion that the most straightforward explanation for the existence of certain mental disor- 
ders is that they have co-evolved as costs of attractive mental traits. Not all mental disorders, however, can be explained in this way.

The telltale signs of a mental disorder that is likely to be a cost associated with a trait used in mate choice are: (a) many of the individuals afflicted are also found to have an attractive mental trait; and (b) the mental trait in question should be comparatively more attractive than other mental traits that have no apparent survival costs associated with them. The broad category of mental disorders generally referred to as schizophrenia is a good candidate in this respect because: (a) its incidence in human males is significantly correlated with creative intelligence, the type of intelligence generally displayed by successful artists, which is an attractive mental trait (MiLlER and TAL 2007; NETTLE 2006b); and (b) creative intelligence is considered to be one of the most attractive mental traits in human males, to the point of females at the peak of their fertility cycles finding creative but poor males significantly more attractive than uncreative but wealthy ones (HASELTON and MILLER 2006).

CRESPI (2006), in a response to a thorough and provocative argument by KELLER and MILLER (2006) regarding the evolutionary bases of mental disorders, makes a point that is similar to the one made above (see, also, NETTLE 2006), and also notes that schizophrenia has a less debilitating effect on human females than males. Ancestral human females, due to their preference for males showing high levels of creative intelligence, might have also selected a co-evolved cost that affects not only males by also the females themselves through gene correlation between the sexes (GILLESPIE 2004; MAYNARD SMITH 1998). Other traits that differentiate females from males, however, may account for differences in the actual survival cost of schizophrenia in females and males. For example, males show a greater propensity toward risk-taking than females (BuSs 1999; MILLER 2000), and schizophrenia may positively moderate the negative relationship between risktaking propensity and survival success.

\section{CONCLUSION}

Several original contributions were made in the preceding sections. A new mathematical framework for reasoning about evolution of traits through selection was presented whereby several important conclusions can be derived by means of simple algebraic operations. The framework combines the Price Equation (PRICE 1970) with the path analysis method (WRIGHT 1934, 1960), by deriving a fundamental requirement for evolution of traits through selection that relies on the relationship between standardized measures of fitness and traits. Costly traits used in mate choice are defined, as part of the mathematical framework, as traits that increase mating success while at the same time decreasing survival success.

The mathematical framework is then used to show how path models can be used by evolutionary psychologists to theorize about the evolution of complex mate 
choice traits, and how theoretical models can be tested with modern humans through the method of path analysis. The mathematical framework is also used in the development of conclusions regarding the evolution of independent traits used in mate choice, the limit on the number of independent traits used in mate choice, the evolution of interdependencies among those traits, and the probability of evolution and relative attractiveness of those traits.

The conclusions stemming from the mathematical analysis are in turn used to develop key exploratory hypotheses, which can be summarized as follows: costly traits used in mate choice by humans should be generally less common and more attractive than non-costly ones, costly traits should be disproportionately less common in human females than males, and certain harmful human mental disorders may have co-evolved as costs of attractive mental traits.

It should be noted that the discussion presented here does not directly address the speed at which a trait may evolve in a population through selection. That speed, often measured in generations, is a function of the non-standardized impact of a trait on fitness (HARTL and CLARK 2007). That impact is the selective advantage that the trait confers on the individuals that possess it, in terms of how many more surviving offspring they generate. The equations included here are expressed in path coefficients, which are essentially standardized coefficients of association. For example, a non-costly trait measure may be strongly associated with fitness if both measures closely approach a line when plotted. But the trait's absolute, as opposed to standardized, impact on fitness may be much lower at a given point in time than that of a costly trait whose association with fitness is less strong. In this case, the costly trait will have the weakest association with fitness of the two and yet may evolve significantly faster.

The reason for the evolution of costly traits used in mate selection presented here differs from that proposed by ZAHAVI (1975), whose core argument is that for animal signals to be reliable they also must be costly (see also: ZAHAVI 2003; ZAHAVI and ZAHAVI 1997). This is the essence of Zahavi's handicap principle, which applies to animal signals in general, of which many traits used in mate choice can be seen as a subclass. There is little doubt that costly traits are indeed useful in mate choice (GRAFEN 1990); this is chiefly due to the fact they are hard to fake. However, the notion that mate choice traits must be costly to be reliable conflicts with a few simple facts: (a) they are a lot less common in nature than non-costly mate choice traits, even though they are presumably more reliable; and (b) they are predominantly observed in males, and rarely in females, even though males also choose females for mating.

The large train displayed by the male in the peacock species has been presented as strong evidence in support of the handicap principle (ZAHAVI and ZAHAVI 1997). Yet, the male peacock also has other traits that are apparently used in mate choice and that are not costly, such as the crest atop its head and the brightly colored feathers on its chest. If the costly trait, namely the train, is the only reliable 
one, it is puzzling that evolution would favor the existence of multiple costly and non-costly traits used for mate choice.

Costly traits used in mate choice appear to be less likely to have evolved, and as likely to have been more attractive than non-costly traits, if and when they evolved. The reason for their rarity and attractiveness is that costly traits must overcome a threshold to evolve, where the threshold is a function of their survival cost. They must be so attractive to the members of the opposite sex that their survival cost is offset by the mating advantage conferred by them. These conclusions are consistent with the fact that the male peacock has more non-costly than costly traits used in mate choice by females. The conclusions are also consistent with the finding by PETRIE et al. (1991) that the train of the male peacock, and especially the number of eye spots on the train, is by far the most attractive trait for the peahens. Those conclusions arguably open the door for a new understanding of the evolution of mate choice traits in various species, including humans, where what is costly to evolve is not necessarily more reliable yet is (in terms of overall fitness) costly not to use.

\section{ACKNOWLEDGEMENTS}

The author would like to thank: the Editor, Daniel Nettle, and the anonymous reviewers; Fernando Quintana, for comments and suggestions on earlier versions of this article; and Texas A\&M International University, for its institutional support. Thanks are also due to those attending the presentation titled "Costly Mate Choice Traits Should be Fairly Rare and Particularly Attractive to Members of the Opposite Sex”, given by the author at the 2008 Annual Meeting of the Human Behavior and Evolution Society, in Kyoto, Japan. Several of the ideas discussed in that presentation formed the basis for this article, and the refinement of those ideas has benefited from the questions and comments received during and after the presentation. All errors and omissions are the sole responsibility of the author.

\section{REFERENCES}

ANDERSON, D.J. (1990): On the evolution of human brood size. Evolution, 44(2), 438-440.

BATEMAN, A.J. (1948): Intra-sexual selection in Drosophila. Heredity, 2(3), 349-368.

BoAz, N.T. \& AlmQuist, A.J. (2001): Biological Anthropology: A Synthetic Approach to Human Evolution. Upper Saddle River, NJ: Prentice Hall.

Buss, D.M. (1995): The Evolution of Desire: Strategies of Human Mating. New York, NY: Basic Books.

Buss, D.M. (1999): Evolutionary Psychology: The New Science of the Mind. Needham Heights, MA: Allyn \& Bacon.

CARTwright, J. (2000): Evolution and Human Behavior: Darwinian Perspectives on Human Nature. Cambridge, MA: The MIT Press.

CHIN, W.W. (2001): PLS-Graph User's Guide. Houston, TX: Soft Modeling Inc. 
COHEN, D.L. \& BELSKY, J. (2008): Individual differences in female mate preferences as a function of attachment and hypothetical ecological conditions. Journal of Evolutionary Psychology, 6(1), 25-42.

Crespi, B. (2006): The natural selection of psychosis. Behavioral and Brain Sciences, 29(4), $410-411$.

Darwin, C.R. (1871): The Descent of Man, and Selection in Relation to Sex. London, England: John Murray.

DuncAn, O.D. (1966): Path analysis: Sociological examples. The American Journal of Sociology, 72(1), 1-16.

FinK, B., Grammer, K. \& ThORnhill, R. (2001): Human (Homo sapiens) facial attractiveness in relation to skin texture and color. Journal of Comparative Psychology, 115(1), 92-99.

FisHER, R.A. (1930): The Genetical Theory of Natural Selection. Oxford, England: Oxford University Press.

FletCHER, J.A. \& ZWICK, M. (2007): The evolution of altruism: Game theory in multilevel selection and inclusive fitness. Journal of Theoretical Biology, 245(1), 26-36.

FoX, C.W. \& WolF, J.B. (eds) (2006): Evolutionary Genetics: Concepts and Case Studies. New York, NY: Oxford University Press.

FrANK, S.A. (1995): George Price's contributions to evolutionary genetics. Journal of Theoretical Biology, 175(3), 373-388.

FRANK, S.A. (1997): The design of adaptive systems: Optimal parameters for variation and selection in learning and development. Journal of Theoretical Biology, 184(1), 31-39.

FuRnHAM, A., SWAmI, V. \& SHAH, K. (2006): Body weight, waist-to-hip ratio and breast size correlates of ratings of attractiveness and health. Personality and Individual Differences, 41(3), 443-454.

GANGestad, S.W. \& THORnhiLl, R. (2003): Facial masculinity and fluctuating asymmetry. Evolution and Human Behavior, 24(4), 231-241.

GILlespie, J.H. (2004): Population Genetics. Baltimore, MD: The Johns Hopkins University Press.

Grafen, A. (1990): Biological signals as handicaps. Journal of Theoretical Biology, 144(4), $517-546$.

Grafen, A. (2002): A first formal link between the Price Equation and an optimization program. Journal of Theoretical Biology, 217(1), 75-91.

Grafen, A. (2006): Optimization of inclusive fitness. Journal of Theoretical Biology, 238(3), 541-563.

Hair, J.F., Black, W.C., Babin, B.J. \& Anderson, R.E. (2009): Multivariate Data Analysis. Upper Saddle River, NJ: Prentice Hall.

HAMiLTON, W.D. \& ZUK, M. (1982): Heritable true fitness and bright birds: A role for parasites? Science, 218(4570), 384-387.

Hartl, D.L. \& Clark, A.G. (2007): Principles of Population Genetics. Sunderland, MA: Sinauer Associates.

HAselton, M. \& MilleR, G.F. (2006): Women's fertility across the cycle increases the shortterm attractiveness of creative intelligence. Human Nature, 17(1), 50-73.

HeNRICH, J. (2004): Cultural group selection, coevolutionary processes and large-scale cooperation. Journal of Economic Behavior \& Organization, 53(1), 3-35.

HILL, K. \& KaPLAN, H. (1999): Life history traits in humans: Theory and empirical studies. Annual Review of Anthropology, 28(1), 397-430.

Iredale, W., Van Vugt, M. \& Dunbar, R. (2008): Showing off in humans: Male generosity as a mating signal. Evolutionary Psychology, 6(3), 386-392. 
KeLLER, M. \& MilLER, G.F. (2006): Which evolutionary genetic models best explain the persistence of common, harmful, heritable mental disorders? Behavioral and Brain Sciences, 29(4), 385-404.

KennY, D.A. (1979): Correlation and Causation. New York, NY: John Wiley \& Sons.

KLINE, R.B. (1998): Principles and Practice of Structural Equation Modeling. New York, NY: The Guilford Press.

KocK, N. (2010): WarpPLS 1.0 User Manual. Laredo, Texas: ScriptWarp Systems.

KокKO, H. (1998): Good genes, old age and life history trade-offs. Evolutionary Ecology, 12(6), 739-750.

KoKko, H., Brooks, R., McNamara, J.M. \& Houston, A.I. (2002): The sexual selection continuum. Proceedings of the Royal Society of London: Biological Sciences, 269(1498), $1331-1340$.

LANDE, R. (1982): A quantitative genetic theory of life history evolution. Ecology, 63(3), 607-615.

LehmanN, L., KelLeR, L.F. \& KOKKO, H. (2007): Mate choice evolution, dominance effects and the maintenance of genetic variation. Journal of Theoretical Biology, 244(2), 282-295.

Lieberman, E., Hauert, C. \& NowAK, M. (2005): Evolutionary dynamics on graphs. Nature, 433(7023), 312-316.

Lyle, H.F. \& Sullivan, R.J. (2007): Competitive status signaling in peer-to-peer file-sharing networks. Evolutionary Psychology, 5(2), 363-382.

MaruyamA, G.M. (1998): Basics of Structural Equation Modeling. Thousand Oaks, CA: Sage Publications.

MAYNARD SMITH, J. (1971): What use is sex? Journal of Theoretical Biology, 30(2), 319-335.

MAYNARD SMith, J. (1994): Must reliable signals always be costly? Animal Behaviour, 47(5), $1115-1120$.

MAYNARD SMith, J. (1998): Evolutionary Genetics. New York, NY: Oxford University Press.

Maynard Smith, J. \& HARPER, D. (2003): Animal Signals. New York, NY: Oxford University Press.

McElreath, R. \& Boyd, R. (2007): Mathematical Models of Social Evolution: A Guide for the Perplexed. Chicago, IL: The University of Chicago Press.

MillaR, M. (2010): Trait inferences about prospective mates: Effects of relationship length and social dominance. Journal Journal of Evolutionary Psychology, 8(3), 245-259.

Miller, G.F. (2000): The Mating Mind: How Sexual Choice Shaped the Evolution of Human Nature. New York, NY: Doubleday.

MiLLER, G.F. (2000b): Sexual selection for indicators of intelligence. In: G. Bock, J. Goode \& K. Webb (eds), The Nature of Intelligence. New York, NY: John Wiley \& Sons, pp. 260-275.

MiLLER, G.F. \& TAL, I. (2007): Schizotypy versus intelligence and openness as predictors of creativity. Schizophrenia Research, 93(1), 317-324.

Mueller, R.O. (1996): Basic Principles of Structural Equation Modeling. New York, NY: Springer.

Nesse, R.M. \& Williams, G.C. (1994): Why We Get Sick: The New Science of Darwinian Medicine. New York, NY: Random House.

NetTle, D. (2006a): Reconciling the mutation-selection balance model with the schizotypycreativity connection. Behavioral and Brain Sciences, 29(4), 418.

NetTle, D. (2006b): Schizotypy and mental health amongst poets, visual artists, and mathematicians. Journal of Research in Personality, 40(6), 876-890.

O’MEARA, T. (2000): Introduction to Quadratic Forms. New York, NY: Springer-Verlag.

PAGE, K.M. \& NOWAK, M.A. (2002): Unifying evolutionary dynamics. Journal of Theoretical Biology, 219(1), 93-98. 
Petrie, M., Halliday, T. \& Sanders, C. (1991): Peahens prefer peacocks with elaborate trains. Animal Behaviour, 41(2), 323-331.

PRICE, G.R. (1970): Selection and covariance. Nature, 227(1), 520-521.

RENCHER, A.C. (1998): Multivariate Statistical Inference and Applications. New York, NY: John Wiley \& Sons.

Rice, S.H. (2004): Evolutionary Theory: Mathematical and Conceptual Foundations. Sunderland, MA: Sinauer Associates.

Rhodes, G., Chan, J., Zebrowitz, L.A. \& Simmons, L.W. (2003): Does sexual dimorphism in human faces signal health? Proceedings of the Royal Society of London: Biology Letters, 270(S1), S93-S95.

Robert, C.P. \& CASElla, G. (2005): Monte Carlo Statistical Methods. New York, NY: Springer.

Rosenthal, R. \& Rosnow, R.L. (1991): Essentials of Behavioral Research: Methods and Data Analysis. Boston, MA: McGraw Hill.

SCHumacker, R.E. \& Lomax, R.G. (2004): A Beginner's Guide to Structural Equation Modeling. Mahwah, NJ: Lawrence Erlbaum.

SHACKELFORD, T.K., SCHMITT, D.P. \& Buss, D.M. (2005): Universal dimensions of human mate preferences. Personality and Individual Differences, 39(2), 447-458.

SinGH, D. \& RANDALL, P.K. (2007): Beauty is in the eye of the plastic surgeon: Waist-hip ratio (WHR) and women's attractiveness. Personality and Individual Differences, 43(2), 329-340.

THORNHILl, R. \& GANGESTAD, S.W. (2006): Facial sexual dimorphism, developmental stability, and susceptibility to disease in men and women. Evolution and Human Behavior, 27(2), 131-144.

VAn Veelen, M. (2005): On the use of the Price Equation. Journal of Theoretical Biology, 237(4), 412-426.

Wells, T.J., Dunn, A.K., Sergeant, M.J.T. \& Davies, M.N.O. (2009): Multiple signals in human mate selection: A review and framework for integrating facial and vocal signals. Journal Journal of Evolutionary Psychology, 7(2), 111-139.

WiLson, E.O. (2000): Sociobiology: The New Synthesis. Cambridge, MA: Harvard University Press.

Wold, S., Trygg, J., Berglund, A. \& AntTi, H. (2001): Some recent developments in PLS modeling. Chemometrics and Intelligent Laboratory Systems, 58(2), 131-150.

WRIGHT, S. (1934): The method of path coefficients. The Annals of Mathematical Statistics, 5(3), $161-215$.

WRIGHT, S. (1960): Path coefficients and path regressions: Alternative or complementary concepts? Biometrics, 16(2), 189-202.

ZAHAVI, A. (1975): Mate selection-A selection for a handicap. Journal of Theoretical Biology, 53(1), 205-214.

ZAHAvi, A. (2003): Indirect selection and individual selection in sociobiology. Animal Behavior, 65(5), 859-863.

Zahavi, A. \& Zahavi, A. (1997): The Handicap Principle: A Missing Piece of Darwin's Puzzle. Oxford, England: Oxford University Press. 


HOME
Institutional Login
Recognized as:
Texas A\&M International
University (776-53-508)
Welcome!
To use the personalized
features of this site, please log
in or register.
If you have forgotten your
username or password, we
can help.

\section{My Profile}

Marked Items

Alerts

Order History

All Saved Items

My Favorites

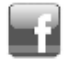

Become a fan

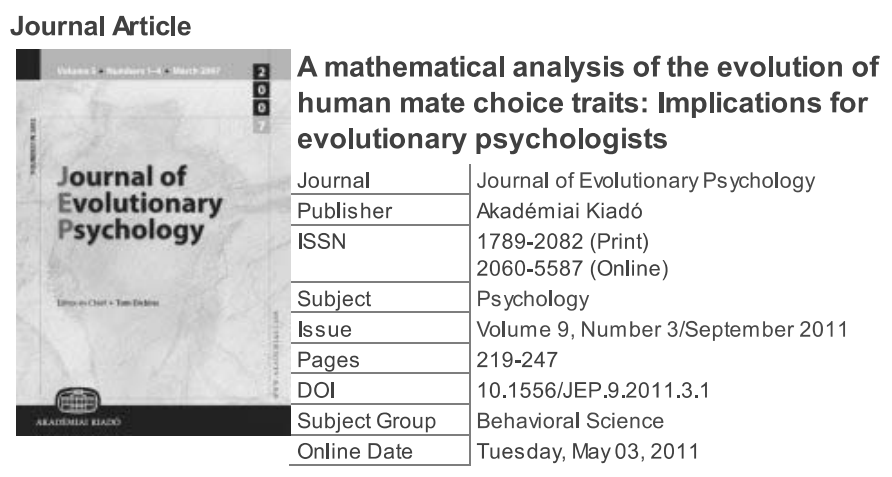

\begin{tabular}{l} 
Find \\
\hline $\begin{array}{l}\text { Within all content } \\
\text { Within this journal } \\
\text { Within this issue }\end{array}$ \\
Export this chapter \\
\hline RIS | Text
\end{tabular}

Authors

Ned Kock ${ }^{1,2}$

${ }^{1}$ Texas A\&M International University Laredo USA

${ }^{2}$ Texas A\&M International University Division of International Bus. and Tech. Studies 5201 University Boulevard Laredo TX78041 USA

\section{Abstract}

Sexually reproducing organis $m s$ often rely on various traits to judge the attractiveness of potential mates. Many mate choice preferences and traits have evolved through selection by those organisms' ancestors, with traits having been either costly (detrimental to survival) or noncostly in the environment of their evolutionary adaptation. A general mathematical analysis of the evolution of traits used in mate choice is presented. The analysis builds on a combination of Price's covariance equation and Wright's method of path analysis, and includes a set of Monte Carlo simulations. The usefulness of the mathematical analysis is demonstrated through the development of a small but important set of hypotheses and implications for the human species: (1) costly traits used in mate choice by humans should be generally less common and more attractive to the other sex than non-costly traits; (2) costly traits should be disproportionately less common in human females than in males; and (3) some harmful human mental disorders, such as schizophrenia, may have coevolved as costs of attractive mental traits. It is also shown that similar analyses can be easily em ployed by evolutionary psychologists to theorize about the evolution of complex mate choice traits, and to test the resulting theories with modern humans through the method of path analysis.

\section{Keywords}

Price equation, path modeling, path analysis, sexual selection, mate choice, costly traits, mental traits, mental disorders, brain evolution

Fulltext Preview (Small, Large)
Add to marked items Add to shopping cart Add to saved items Recommend this article 